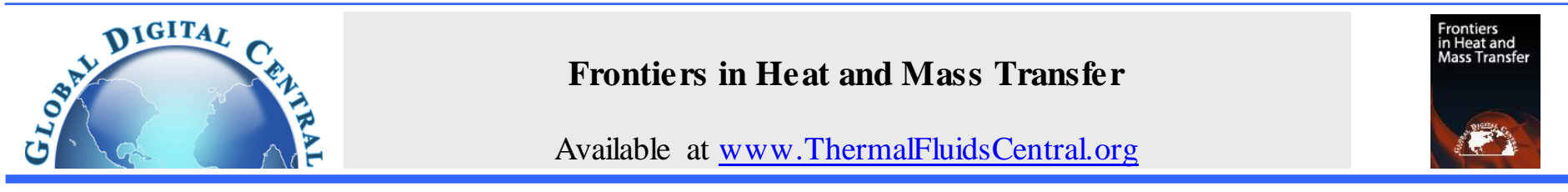

\title{
SQUEEZE FILM LUBRICATION OF ASYMMETRIC ROLLERS BY BINGHAM PLASTIC FLUID
}

\author{
Revathi Gadamsetty $^{\mathrm{a},{ }^{*}}{ }^{\dagger}$, Venkata Subrahmanyam Sajja ${ }^{\mathrm{b}}$, P. Sudam Sekhar ${ }^{\mathrm{c}}$, Dhaneshwar Prasad ${ }^{\mathrm{d}}$ \\ ${ }^{a}$ Research Scholar, Department of Mathematics, Koneru Lakshmaiah Education Foundation, Guntur-522502, Andhra Pradesh, India \\ ${ }^{b}$ Department of Mathematics, Koneru Lakshmaiah Education Foundation, Guntur-522502, Andhra Pradesh, India \\ ${ }^{c}$ Division of Mathematics, Vignan's Foundation for Science, Technology and Research, Guntur-522231, Andhra Pradesh, India \\ ${ }^{d}$ Department of Mathematics, Kanchi Mamunivar Government Institute for Post Graduate Studies and Research, Puducherry-605008, India
}

\begin{abstract}
An attempt has been made to investigate hydrodynamic lubrication characteristics of asymmetric roller bearings lubricated by thin fluid film under the operating behavior of line contact for a heavily loaded rigid system for normal squeezing motion with cavitation points. The lubricant follows non-Newtonian incompressible Bingham plastic fluid model where the fluid viscosity is supposed to vary with hydrodynamic pressure. The equations which govern the fluid flow such as continuity and momentum equation are solved first analytically and later numerically using MATLAB. The numerical results are achieved for the velocity, pressure, load, and traction forces by varying different physical parameters like rolling ratio, squeezing parameter, and yield stress parameter. The obtained results are investigated numerically and graphically. Finally, it is concluded that a notable change is observed in velocity, pressure, load and traction with Newtonian and non-Newtonian fluids. Results follow good agreement with available literature.
\end{abstract}

Keywords: Hydrodynamic lubrication, Non-Newtonian, Bingham plastic, Squeezing, Incompressible, Viscosity.

\section{INTRODUCTION}

The squeeze film lubrication process is of great importance in many engineering fields and is normally found in the course of motors, plane motors, machine devices, super gadget, and skeletal joints. Expectedly, the forecast of squeeze film movement recognizes that the salve goes probably as a viscous Newtonian fluid. Precisely, the forecast of squeeze film motion assumes that the lubricant acts as a Newtonian viscous fluid. However, experimental results show that the inclusion of small quantity of long-chained additives to a Newtonian fluid reduces the sensitivity of the lubricant to change in shear rate and dispenses beneficial effects on the load-carrying and frictional characteristics (Prasad et al., 2012).

Hydrodynamic lubrication is a strategy which is utilized to diminish friction and wear of scouring surfaces with fluid. The aim of hydrodynamic lubrication is typically to add a proper liquid, with the goal that it enters into the contact zone between scouring surface and makes a fluid thin film. This film separates the surfaces from contacting one another and for the most part of it diminishes friction and wear (Revathi et al., 2020).

Bearing are consistently reliant upon unbelievably profound loads, top speeds and heavy slip conditions. The effect of the high load on the treatment in the concentrated contact causes high pressure age in the fluid film. In the high pressure territory, material property of the oil, particularly viscosity, isn't, now consistent yet changes continually with pressure and temperature (Prasad et al. 2012).

Prasad et al. (1987) introduced a new approach which manages the issue of a couple of inelastic intensely highly fitted rollers with rolling and squeezing motions, greased up by power-law liquid accepting the fluid consistency to change dramatically with temperature and pressure.
At the same time, it was processed mathematically by settling this issue with adjusted Reynolds and energy conditions. Patrice Estellé \& Christophe Lanos (2007) derived an analytical solution of a Bingham plastic squeeze film flow with friction at the plates under slip in a particular zone. The result was determined accepting the sample gap to be little when contrasted with the plate radius and from the rate of flow energy scattering. Prasad et al. (2012) studied a journal bearing problem with non-Newtonian lubrication considering thermal and squeezing effects. An appreciable change in pressure and temperature was observed. Also, a non-Newtonian fluid was considered by highlighting the importance of the work due to non-dimensional scheme for pressure, temperature and consistency. Prasad et al. (2014) investigated another hydrodynamic problem with rigid system of roller bearings, greased up by a flimsy compressible liquid for normal squeezing movement that focused on expecting the consistency and density of the fluid to differ corresponding to the temperature and the pressure.

In modern engineering, many fluids show non-Newtonian behavior, therefore many researchers are more interested in those industrial non-Newtonian fluids and their dynamics (Banerjee et al., 2018). The exhibited qualities of non-Newtonian Bingham plastic fluid flow are used to depict the way of fluids, explicitly, fluids for quite a while; and besides they are used to portray the progression of melts and slurries in molds (Christopher and Tichy, 1992). Speculative examinations of grease with Bingham model return to Milne (1954). He investigated the basic 1-D journal and slider bearings, and infers inflexible 'cores' potentially added to either surfaces. Besides, Christopher Dorier and John Tichy presented a model of the way of Bingham-like fluids which show a yield stress (Christopher and Tichy, 1992). Recently, Revathi et al. (2019) studied non-Newtonian

* Currently in Department of Mathematics, Gokaraju Rangaraju Institute of Engineering and Technology, Hyderabad, India.

Corresponding author. Email: grevathi1331@gmail.com 
lubrication of asymmetric rollers for highly stacked rigid system with incompressible Bingham plastic fluid in rolling/sliding line contact, considering the fluid viscosity to vary with the hydrodynamic pressure. The fluid velocity trends were obtained and the results, particularly, pressure, load and traction forces were found to agree with available results. Further, Revathi et al. (2020) investigated a similar problem on lubrication characteristics of anti-symmetric rollers using incompressible Bingham plastic fluid. Results particularly temperatures, pressure, including traction and load were in good agreement with available results.

In view of above discussion, the purpose of this paper is to study the squeezing effects in hydrodynamic lubrication of asymmetric rollers by an incompressible Bingham plastic fluid for heavily loaded rigid rolling/sliding line contact problem assuming the viscosity of the lubricant to be varied with hydrodynamic pressure; the rolling ratios are used to study the rolling/sliding effects of surfaces on pressure, load and traction.

\section{THEORETICAL MODEL}

The system in this paper is considered in such a way that both the surfaces are having same radius but moving with different velocities. Further it can be noted that the upper surface is moving faster than lower surface. The complete flow configuration is shown in Fig. (1).

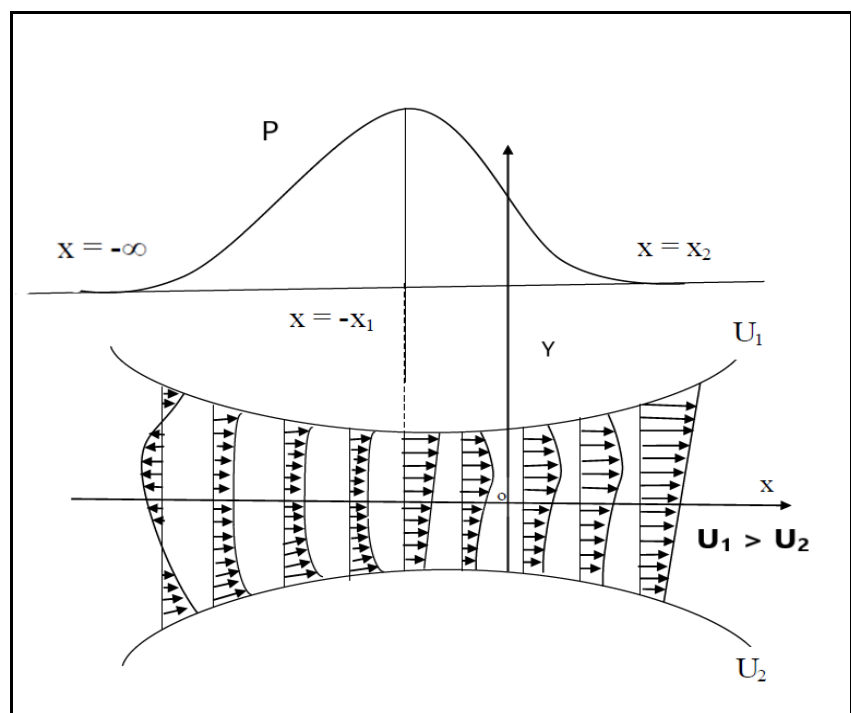

Fig. 1 Lubrication of Asymmetric Rollers

\subsection{Mathematical formulation}

The equations such as continuity and momentum which govern the incompressible fluid flow are considered under usual assumptions (Prasad and Singh, 1987) as follows:

$$
\begin{aligned}
& \frac{\partial u}{\partial x}+\frac{\partial v}{\partial y}=0 \\
& \frac{d p}{d x}=\frac{\partial \tau}{\partial y}
\end{aligned}
$$

where ' $p$ ' and ' $\tau$ ' are hydrodynamic pressure and shear stress of the fluid respectively. The constitutive equation for Bingham plastic fluid is given by Sasaki et al. (1962)

$$
\tau= \pm \tau_{0}+\mu \frac{\partial u}{\partial y}
$$

where $\mu$ is the viscosity of the fluid taken by $\mu=\mu_{0} e^{\alpha p}$

and the equation for thickness of the film is to be $h=h_{0}+\frac{x^{2}}{2 R}$

$\mathrm{R}$, being the radius of the equivalent cylinder.

\subsection{Boundary conditions}

The boundary conditions for this problem at both upper and lowers surfaces are taken as

$u=U_{1}$ and $v=\frac{V}{2}+U_{1} \frac{d h}{d x}$ at $y=h$
$u=U_{2}$ and $v=-\frac{V}{2}-U_{2} \frac{d h}{d x}$ at $y=-h$

$p=0$ at $x=-\infty$

$p=0$ and $\frac{d p}{d x}=0$ at $x=x_{2}$

where $U_{1}$ and $U_{2}$ are velocities of the rolling cylinders as shown in Fig.(1).

Integration of the equation (2) using the above boundary conditions gives the fluid velocity expression as below

$u=\frac{3\left(y^{2}-h^{2}\right)\left[\left(U_{1}+U_{2}\right)\left(h-h_{1}\right)+V\left(x+x_{1}\right)\right]}{4 h^{3}}+\frac{y}{2 h}\left(U_{1}-U_{2}\right)+\frac{1}{2}\left(U_{1}+U_{2}\right)$

Now the volume flux ' $Q$ ' for the fluid flow can be obtained by integrating the velocity within the gap between the surfaces is presented below

$Q=\int_{-h}^{h} u d y=h\left(U_{1}+U_{2}\right)-\frac{2 h^{3}}{3 \mu} \frac{d p}{d x}$

And the volume flux at the point of maximum pressure is

$Q\left(-x_{1}\right)=\left(U_{1}+U_{2}\right) h_{1}$

Where the film thickness $h_{1}$ at $x=-x_{1}$ is considered to be $h_{1}=1+x_{1}{ }^{2}$

\subsection{Reynolds equation}

Solving the equation (2) using the boundary conditions gives the pressure Reynolds equation which is presented below

$\frac{d p}{d x}=\frac{3 \mu}{2 h^{3}}\left[\left(U_{1}+U_{2}\right)\left(h-h_{1}\right)+V\left(x+x_{1}\right)\right]$

\subsection{Dimensionless scheme}

The following dimensionless scheme for roller bearings is applied throughout this paper

$$
\begin{aligned}
& \bar{x}=\frac{x}{R}, \quad \bar{p}=\alpha p, \quad \overline{\mathrm{h}}=1+\bar{x}, \bar{\mu}=\overline{\mu_{0}} e^{\bar{p}}, \\
& \overline{\tau_{0}}=\frac{\alpha R \tau_{0}}{h_{0}}, \overline{\mu_{0}}=\frac{2 \alpha R U}{h_{0}{ }^{2}} \mu_{0}, \overline{\mathrm{U}}=U_{1} / U_{2}
\end{aligned}
$$

The velocity expression and pressure Reynolds equation are written in dimensionless form using the above mentioned dimensionless scheme.

$$
\begin{aligned}
& \bar{u}=\frac{3\left(\bar{y}^{2}-\bar{h}^{2}\right)}{4 \bar{h}^{3}}\left[(1+\bar{U})\left(\bar{h}-\overline{h_{1}}\right)+\bar{V}\left(\bar{x}+\overline{x_{1}}\right)\right]+\frac{(\bar{y}+\bar{h}) \bar{U}+(\bar{h}-\bar{y})}{2 \bar{h}} \\
& \frac{d \bar{p}}{d \bar{x}}=\frac{\left.3 \bar{\mu} \mid(1+\bar{U})\left(\bar{h}-\overline{h_{1}}\right)+\bar{V}\left(\bar{x}+\overline{x_{1}}\right)\right]}{4 \bar{h}^{3}}
\end{aligned}
$$

2.5 Load and Traction (Subrahmanyam and Prasad, 2015)

Since load capacity produces complete estimation of the bearings efficiency, so load capacity is the one of the significant qualities. Hence, Integration of the pressure across the film thickness gives the $\mathrm{x}$ component of the load $\mathrm{W}_{\mathrm{x}}$ per unit length of the cylinder as

$W_{x}=\int_{-h}^{h} p d h$

The dimensionless normal load $\overline{W_{y}}$ is given by

$\bar{W}_{x}=\int_{-\infty}^{\bar{x}_{2}} \bar{x}^{2} \frac{d \bar{p}}{d \bar{x}} d \bar{x}$

Similarly, the normal load carrying capacity Wy can be obtained as

$W_{y}=\int_{-\infty}^{x_{2}} p d x$

The dimensionless normal load $\overline{W_{y}}$ is given by

$\bar{W}_{y}=-\int_{-\infty}^{\bar{x}_{2}} \bar{p} d \bar{x}$

Further, Integration of the shear stress $\tau$ for the entire length gives the traction force $T_{F}$ at the surfaces can be obtained as 
$T_{F h-}=-\int_{-\infty}^{x_{2}} \tau_{y=-h} d x ;$ and $T_{F h+}=-\int_{-\infty}^{x_{2}} \tau_{y=h} d x$

Dimensionless tractions are

$\bar{T}_{F h-}=-\int_{-\infty}^{\bar{x}_{2}} \bar{\tau}_{\bar{y}=-\bar{h}} d \bar{x} ;$ and $\bar{T}_{F h+}=-\int_{-\infty}^{\bar{x}_{2}} \bar{\tau} \bar{y}=\bar{h} d \bar{x}$

\section{RESULTS AND DISCUSSION}

Numerical computations are performed with the following values in this problem:

$U_{2}=400 \mathrm{~cm} / \mathrm{s}, h_{0}=4 \times 10^{-4} \mathrm{~cm}, \alpha=1.6 \times 10^{-9}$ dyne $e^{-1} \mathrm{~cm}^{2}, R=3 \mathrm{~cm}$

\subsection{Velocity profile}

Velocity $\bar{u}$ of the fluid is calculated and presented in Figs.(2-4) respectively for the regions before, after, and at the point of maximum pressure. The profiles in first two graphs look like parabolas having vertices upward and downward directions in the regions before and after the point of maximum pressure.

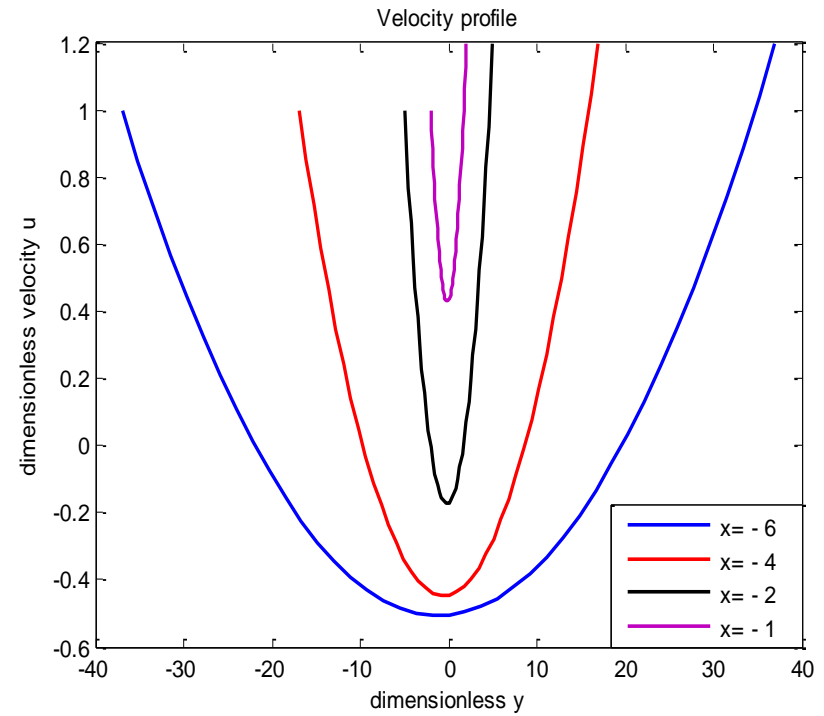

Fig. 2 Velocity Profile

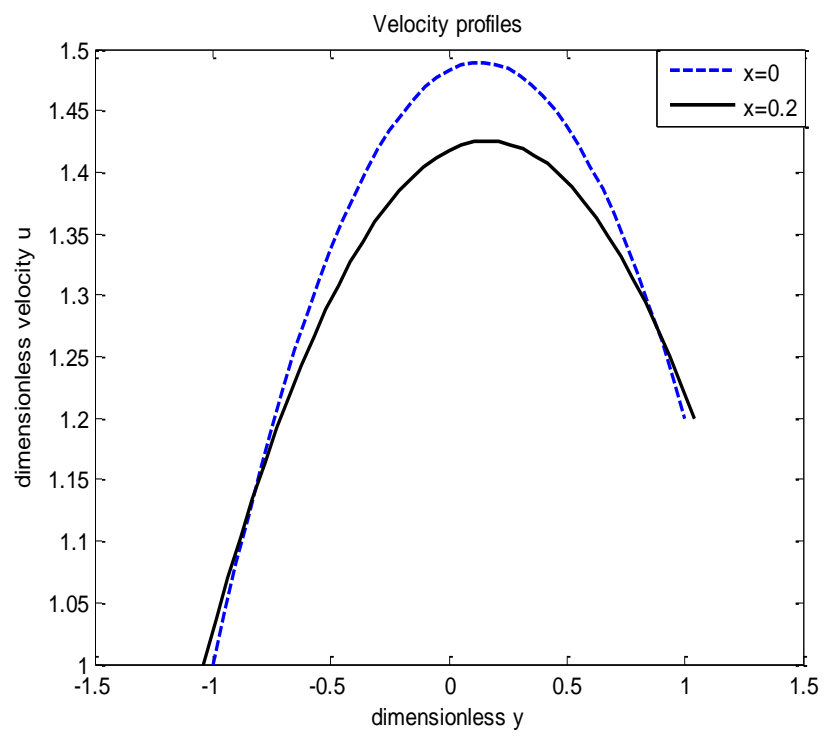

Fig. 3 Velocity profile

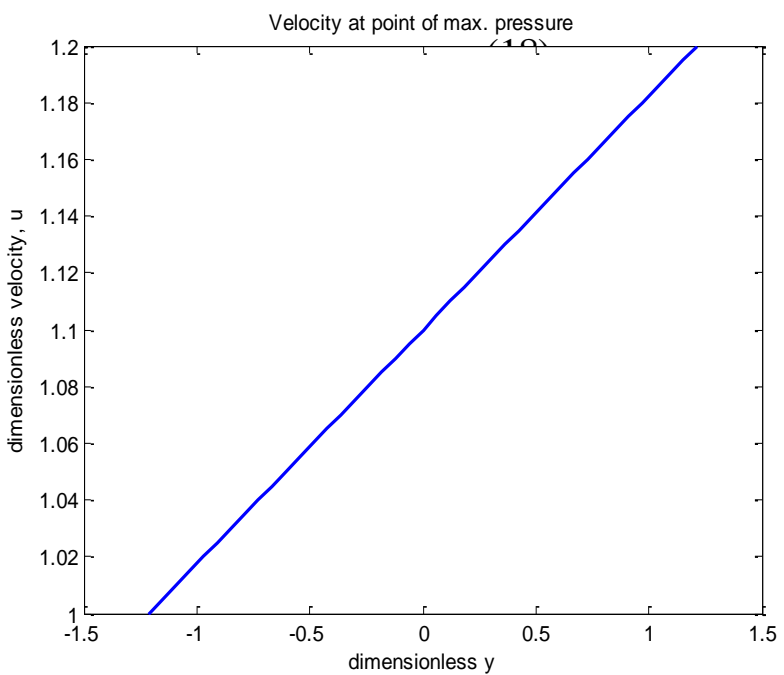

Fig. 4 Velocity profile at point of maximum pressure

The vertices below the $\bar{y}$ line show there is a back flow near the inlet as presented in Fig.(2). Reverse flow was also shown by Prasad and Subrahmanyam (2016). As the fluid progresses, the back flow is over (Prasad and Subrahmanyam, (2016); Revathi et al, (2020); Prasada et al. (1991). But the velocity profile, at the point of maximum pressure, seems to be linearly increasing (Prasad and Subrahmanyam, 2016) and can be observed in Fig. (4).

\subsection{Pressure profile}

The pressure $\bar{p}$ distributions are calculated numerically and presented in Figs. (5-8). It can be observed from Fig. (5) and Fig. (6) that $\bar{p}$ increases with rolling ratio $\bar{U}$ in both the cases with and without squeezing. This indicates that hydrodynamic pressure is more for sliding case than pure rolling. This kind of behavior was observed in Prasada et al., (2012); Prasad and Subrahmanyam, (2016); Revathi et al., (2019); Subrahmanyam and Prasad, (2015). Further, the lubricant pressure for different values of squeezing parameter $\bar{V}$ for pure rolling and sliding case is presented in Fig. (7) and Fig. (8) respectively and can be seen that the pressure decreases with the increasing value of $\bar{V}$. The trend is matching with the results of Prasad et al. (2014).

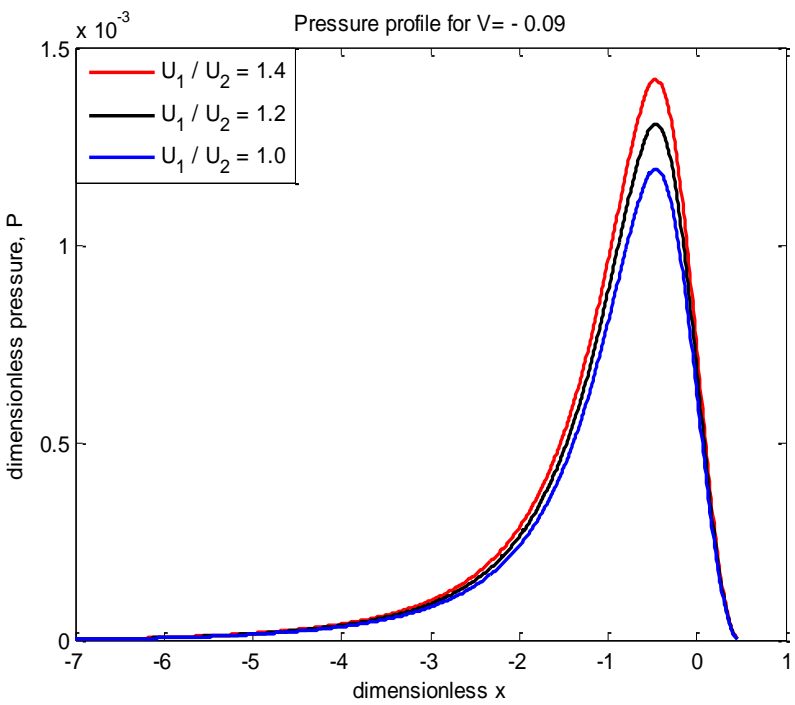

Fig. 5 Pressure profile for different $\bar{U}$ 


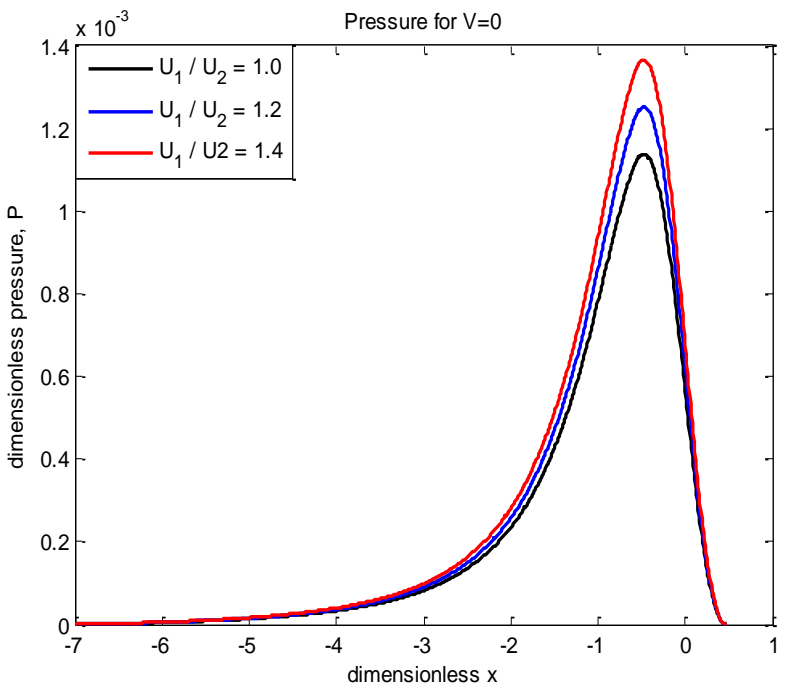

Fig. 6 Pressure profile for different $\bar{U}$

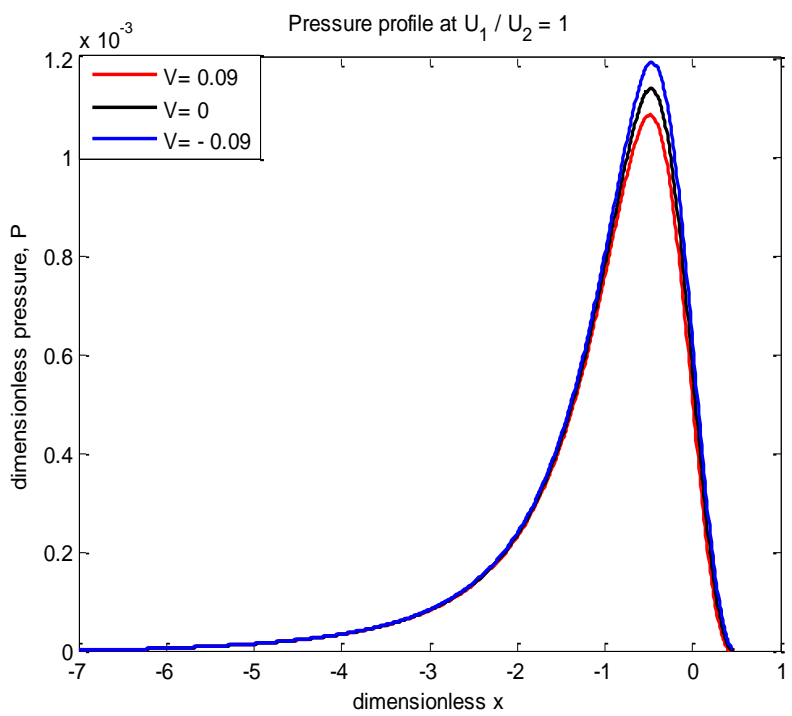

Fig. 7 Pressure profile for pure rolling case

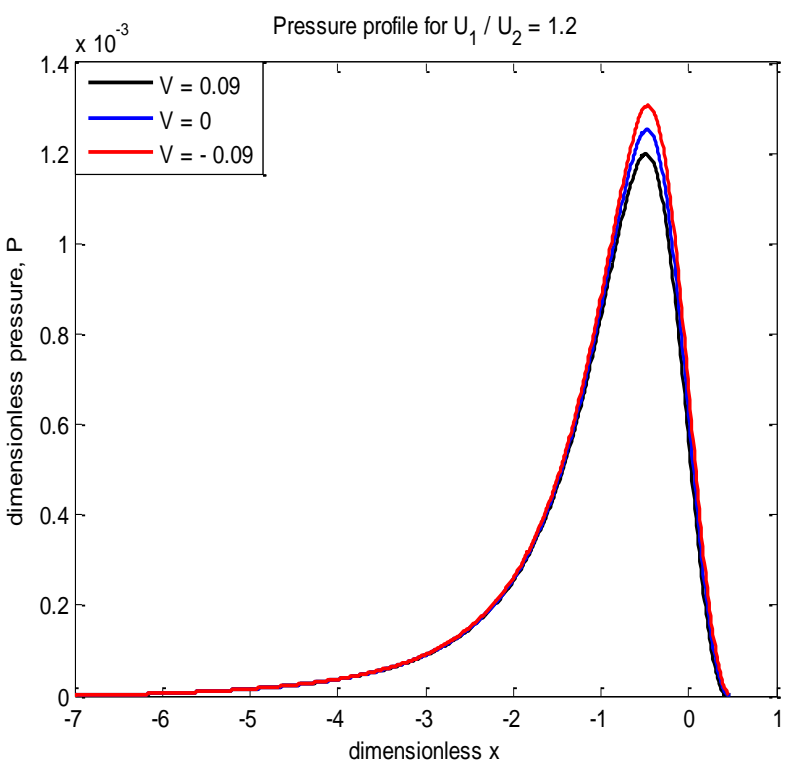

Fig. 8 Pressure profile for sliding case

\subsection{Viscosity $(\bar{\mu})$ Profile}

The lubricant viscosity $\bar{\mu}$ is computed numerically for different values of $\bar{U}$ and $\bar{V}$ and depicted in Fig. (9) and Fig. (10). The lubricant viscosity for different values of squeezing parameter for rolling/sliding case is presented in Fig. (9) and can be observed that the viscosity has impact in the minimum gap between the rollers. Further, Fig. (10) shows that viscosity profile for different values of rolling ratio when $\bar{V}=-0.09$. From this figure one can observe that viscosity increases as rolling ratio increases. The same trend can be observed in Subrahmanyam and Prasad (2015).

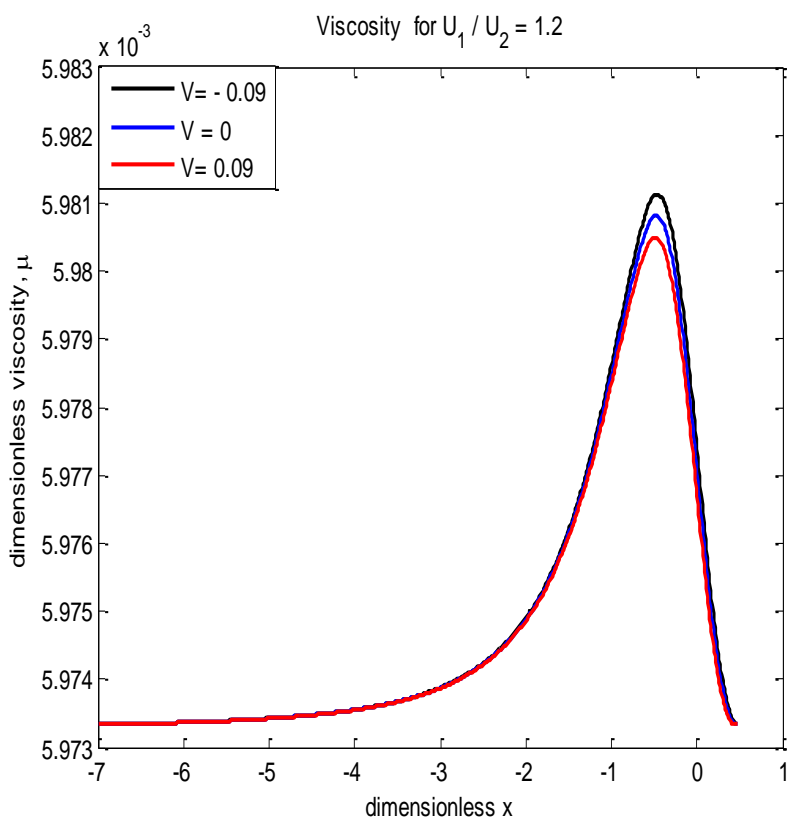

Fig. 9 Viscosity profile

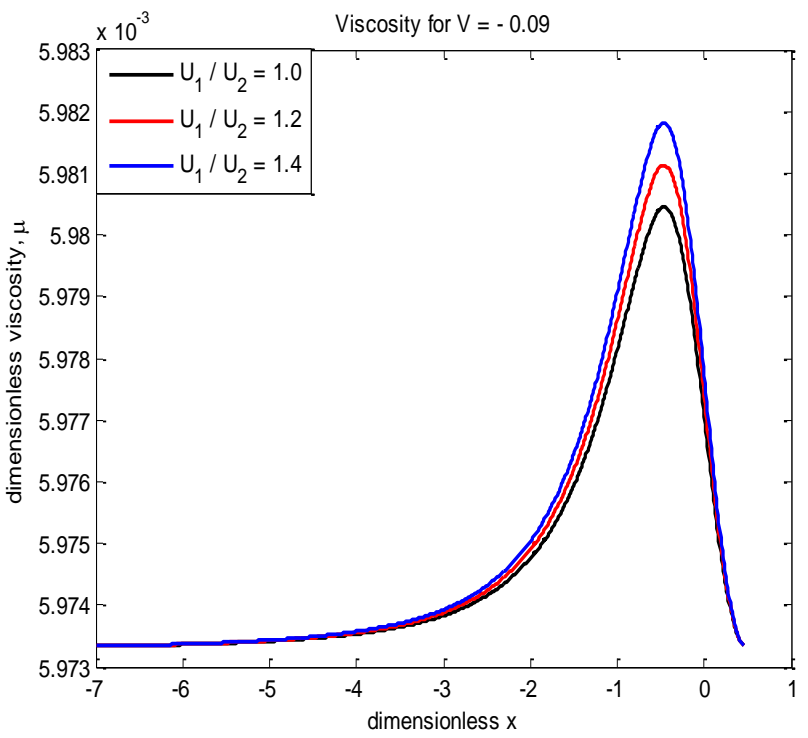

Fig. 10 Viscosity profile

\subsection{Load and Traction}

The dimensionless load values in both $\mathrm{x}$ and $\mathrm{y}$-direction are numerically computed for different values of rolling ratio and squeezing parameters and presented in Table-1. The table shows that load increases in both directions with rolling ratio $\bar{U}$ for fixed value of $\bar{V}$. These results are in good agreement with earlier published results. Prasad et al., (1988); 
Prasad et al., (1991); Prasad and Subrahmanyam, (2016). Further, the load decreases in both directions as squeezing parameter increases for fixed value of $\bar{U}$.

Table 1 Load values

\begin{tabular}{|c|c|c|c|}
\hline $\bar{U}$ & $\bar{V}$ & $\overline{W_{x}}$ & $\overline{W_{y}}$ \\
\hline \multirow{4}{*}{1.0} & -0.09 & 0.00368744 & 0.00181834 \\
\cline { 2 - 4 } & 0 & 0.00360905 & 0.00174268 \\
\cline { 2 - 4 } 1.1 & 0.09 & 0.00353136 & 0.00166697 \\
\hline \multirow{4}{*}{1.2} & -0.09 & 0.00386794 & 0.00190549 \\
\cline { 2 - 4 } & 0 & 0.00378955 & 0.00182984 \\
\cline { 2 - 4 } & 0.09 & 0.00371182 & 0.001754061 \\
\cline { 2 - 4 } & -0.09 & 0.00404845 & 0.00199264 \\
\hline \multirow{3}{*}{1.3} & 0.09 & 0.00397005 & 0.00191701 \\
\cline { 2 - 4 } & -0.09 & 0.00422896 & 0.00207981 \\
\cline { 2 - 4 } & 0.09 & 0.00415056 & 0.00200419 \\
\hline \multirow{3}{*}{1.4} & -0.09 & 0.00407279 & 0.00192831 \\
\cline { 2 - 4 } & 0 & 0.00433107 & 0.00209136 \\
\cline { 2 - 4 } & 0.09 & 0.00425328 & 0.00201544 \\
\hline
\end{tabular}

The traction forces $\bar{T}_{F h}$ have been compute and presented in table- 2 and table-3 at both the lower and upper surfaces for distinct values of $\bar{\tau}_{0}$ and $\bar{U}$. Here $\bar{\tau}_{0}=0$ represents Newtonian case and $\bar{\tau}_{0}>0$ represents nonNewtonian case. The Traction forces increase for fixed value of $\bar{U}$ at both the lower and upper surfaces with $\bar{\tau}_{0}$. Further, the increase of traction forces with $\bar{U}$ at upper surface can be observed from table-3 and indicates that the surface moving with more velocity will have more traction force. These findings are very similar to the results published in Prasad et al., (1988); Prasad et al., (1991); Prasad and Subrahmanyam, (2016). Both the surfaces have the same traction force when $\bar{U}=1$.

Table 2 Traction force values at lower surface

\begin{tabular}{|c|c|c|c|}
\hline $\bar{U}$ & $\bar{\tau}_{0}=0$ & $\bar{\tau}_{0}=0.5$ & $\bar{\tau}_{0}=1$ \\
\hline 1.0 & 0.00372958 & 2.80872958 & 5.61372958 \\
\hline 1.1 & 0.00363405 & 2.80863405 & 5.61363405 \\
\hline 1.2 & 0.00353852 & 2.80853852 & 5.61353852 \\
\hline 1.3 & 0.00344298 & 2.80844298 & 5.61344298 \\
\hline 1.4 & 0.00334744 & 2.80834744 & 5.61334744 \\
\hline 1.5 & 0.00325190 & 2.80825190 & 5.61325190 \\
\hline
\end{tabular}

Table 3 Traction force values at upper surface

\begin{tabular}{|c|c|c|c|}
\hline $\bar{U}$ & $\bar{\tau}_{0}=0$ & $\bar{\tau}_{0}=0.5$ & $\bar{\tau}_{0}=1$ \\
\hline 1.0 & 0.00372958 & 2.80872958 & 5.61372958 \\
\hline 1.1 & 0.00418715 & 2.80918715 & 5.61418715 \\
\hline 1.2 & 0.00464471 & 2.80964471 & 5.61464471 \\
\hline 1.3 & 0.00510227 & 2.81010227 & 5.61510227 \\
\hline 1.4 & 0.00555982 & 2.81055982 & 5.61555982 \\
\hline 1.5 & 0.00601738 & 2.81101738 & 5.61601738 \\
\hline
\end{tabular}

\section{CONCLUSION}

An attempt is made to study the squeeze film lubrication characteristics of line contact problem by an incompressible non-Newtonian Bingham plastic fluid. The continuity and momentum equations both are solved for pressure and the fluid velocity for different values of $\bar{\tau}_{0}$ and the sliding parameter $\overline{\mathrm{U}}$. The following points may be obtained from the results of this work:

- Both the velocity and pressure of the lubricant are independent of $\bar{\tau}_{0}$

- Velocity of the lubricant at point of maximum pressure increases linearly.

- The increase in rolling ratio $\bar{U}$ causes the rise in pressure with and without squeezing.

- Pressure increases with decreasing value of squeezing parameter for pure rolling case.

- The increase in rolling ratio causes increase of load in both $\mathrm{x}$ and y-directions.

- The traction at the upper surface is higher than that of the lower surface due to more speed of upper surface.

- The increase in $\bar{\tau}_{0}$ causes the increase in traction forces at both the surfaces.

\section{NOMENCLATURE}

$\begin{array}{ll}\mathrm{p} & \text { Hydrodynamic pressure } \\ \mathrm{T}_{\mathrm{Fh}} & \text { Traction force } \\ \mathrm{u} & \text { Fluid velocity in } \mathrm{x} \text {-direction } \\ \mathrm{v} & \text { Fluid velocity in y-direction } \\ \mathrm{x}_{1} & \text { Point of maximum pressure } \\ \mathrm{x}_{2} & \text { Point of cavitation } \\ \mathrm{h} & \text { Film thickness } \\ \mathrm{h}_{0} & \text { Minimum film thickness } \\ \alpha & \text { Pressure coefficient } \\ \mathrm{U}_{1}, \mathrm{U}_{2} & \text { Velocities of the surfaces }\end{array}$

\section{REFERENCES}

Astick Banerjee, Aurang Zaib, Krishnendu Bhattacharyya, Mahato, S.K., 2018, "MHD mixed convection flow of a non-Newtonian PowellErying fluid over a permeable exponentially shrinking sheet", Frontiers in Heat and Mass Transfer, 10(30), 1-8.

http://doi.org/10.5098/hmt.10.30

Christopher Dorier and John Tichy, 1992, "Behavior of Bingham-like viscous fluid in lubrication flows, Journal of Non Newtonian fluid mechanics, 45(3), 291-310.

https://doi.org/10.1016/0377-0257(92)80065-6

Dhaneshwar Prasad \& Venkata Subrahmanyam Sajja, 2016, "NonNewtonian Lubrication of Asymmetric Rollers with Thermal and Inertia Effects", Tribology Transactions, 59(5), 818-830,

https://doi.org/10.1080/10402004.2015.1107927

Dhaneshwar Prasad and Punyatma Singh, 1987, “Thermal and squeezing effects in non-Newtonian fluid film lubrication of rollers:, Wear, 119, 175-190.

https://doi.org/10.1016/0043-1648(87)90107-4

Dhaneshwar Prasad, S.V. Subrahmanyam, Sudam Sekhar Panda, 2012, "Thermal Effects In Hydrodynamic Lubrication Of Asymmetric Rollers Using Runge Kutta Fehlberg Method", International Journal of Engineering Science \& Advanced Technology, 2(3), 422-437.

Dhaneshwar Prasad, S.V. Subrahmanyam, Sudam Sekhar Panda, 2014, "Thermal, Squeezing and Compressibility Effects in Lubrication of Asymmetric Rollers”, Tribology in Industry, 36(3), 244-258. 
Dhaneshwar Prasad, Sudam Sekhar Panda, S.V. Subrahmanyam, 2012, "Non-Newtonian Squeeze Film Lubrication of Journal Bearing with Temperature Effect", International Journal of Engineering Science \& Advanced Technology, 2(3), 438-444.

Dhaneshwar Prasad, Venkata Subrahmanyam Sajja, 2016, “Thermal Effects in Non-Newtonian Lubrication of Asymmetric Rollers under adiabatic and Isothermal Boundaries", International Journal of Chemical Sciences, 14(3), 1641-1656.

Gadamsetty Revathi, Venkata Subrahmanyam Sajja, Dhaneshwar Prasad, 2019, "Bingham P lastic Fluid Film Lubrication of Asymmetric Rollers", International Journal of Scientific \& Technology Research, 8(11), 2549-2554.

Gadamsetty Revathi, Venkata Subrahmanyam Sajja, Dhaneshwar Prasad, 2019, "Thermal Effects in Power-law fluid film lubrication of Rolling/Sliding Line Contact", International Journal of Innovative Technology and Exploring Engineering, 8(9), 277-283. http://doi.org/10.35940/ijitee.H7195.078919

Milne, A.A., 1954, “A theory of rheodynamic lubrication”, Kolloidk-Z, 139 Haft $1 / 2,96-101$.

Patrice Estellé, Christophe Lanos, 2007, "Squeeze flow of Bingham fluids under slip with friction boundary condition”, Rheol Acta, 46(3), 397-404. http://doi.org/10.1007/s00397-006-0129-8

Prasad D., Shukla J.B., Singh P., Sinha P., Chhabra R.P., 1991, "Thermal Effects in Lubrication of Asymmetrical Rollers", Tribology
International, 24(4), 239-246. https://doi.org/10.1016/0301-679X(91)90050-J

Prasad, D., Singh, P., Sinha, P., 1998, "Non-Uniform Temperature in Non-Newtonian Compressible Fluid Film Lubrication of Rollers", Journal of Tribology, 110, 653-658. https://doi.org/10.1115/1.3261708

Revathi Gadamsetty ,VenkataSubrahmanyam Sajja, Swetha Lanka, Dhaneshwar Prasad, 2020, "Thermohydrodynamic Lubrication of Asymmetric Rollers by Bingham Plastic Fluid", Solid State Technology, 63(5), 6165-6181.

Revathi Gadamsetty, Venkata Subrahmanyam Sajja, P. Sudam Sekhar, Dhaneshwar Prasad, "Thermal Effects in Bingham Plastic Fluid Film Lubrication of Asymmetric Rollers", Frontiers in Heat and Mass Transfer, 2020, 15(18), 1-7.

http://doi.org/10.5098/hmt.15.18

Subrahmanyam, S. V., and Dhaneshwar Prasad, 2015, "Characterization of Lubrication of Asymmetric Rollers including Subrahmanyam, S. V., Dhaneshwar Prasad, 2015, "Characterization of lubrication of asymmetric rollers including Thermal Effects", Industrial Lubrication and Tribology, 67(3), 246-255. https://doi.org/10.1108/ILT-04-2013-0048

Tokio Sasaki, Haruo Mori, Norio Okino, 1962, "Fluid Lubrication theory of roller bearing", Journal of Basic Engineering, 84(1), 166-174. $\underline{\text { https://doi.org/10.1115/1.3657240 }}$ 